\title{
LEARNING DISABILITY IN CHILDHOOD-ONSET EPILEPSY
}

The occurrence of learning disability (LD) in adults with childhood-onset epilepsy and its impact on medical and social outcome were analyzed in a study at the University of Turku, Finland. The study group of 242 patients included all children aged less than 16 years having active epilepsy, resident in a geographically defined area of the University Hospital in 1964, and followed for 45 years. Data were retrospective for the first 10 years and then prospective by examination every $5^{\text {th }}$ year for the last 35 years of follow-up. LD was present in $182(76 \%)$ patients and was related to the IQ level. LD occurred in $57 \%$ of subjects with IQ $>85$, and in $67 \%$ of those with IQ of $71-85 ; 51 \%$ of patients with LD were mentally retarded. Of the total subjects, $78 \%$ attended regular classes, $12 \%$ attended special classes at ordinary schools, and $9 \%$ attended training schools for mentally handicapped. Mentally nearnormal subjects had hyperkinetic behavior more often than mentally normal subjects ( $44 \%$ vs $8 \%, \mathrm{P}<0.0001$ ). Reading, writing, and speech problems in mentally nonretarded patients occurred in 19,19, and $40 \%$, respectively. Reading disability occurred less often in patients with rolandic epilepsy than with other epilepsies. Speech problems were associated with temporal lobe and minor motor epilepsies. A symptomatic etiology of epilepsy was the only predictor of LD in mentally nonretarded patients. LD and seizure outcome were closely related. A 5-year or longer seizure-free period was achieved in $90 \%$ of patients with no LD compared to $70 \%$ with LD and in $54 \%$ of mentally retarded subjects. Patients with LD or MR relapsed more often than non-LD or nonretarded patients. Risk factors for MR and LD were the occurrence of cerebral palsy, onset of epilepsy before age 6 years, and early resistance to antiepileptic drugs. The degree of LD significantly impacted medical, social, and educational long-term outcomes. (Sillanpaa M. Learning disability: occurrence and long-term consequences in childhood-onset epilepsy. Epilepsy \& Behavior Dec 2004;5:937-944). (Respond: Dr Matti Sillanpaa, Departments of Child Neurology and Public Health, University of Turku, Turku, Finland).

COMMENT. Learning disability is a common complication of childhood-onset epilepsy and it adversely affects medical, social, and educational outcomes. Neurophysiological mechanisms involved in epilepsy that may interfere with learning include the following: 1) disturbance of attention to incoming information, its storage or retrieval during seizures; 2) damage to neural tissue: 3 ) antiepileptic drug toxicity; 4) cognitive impairment related to subclinical generalized spike wave discharges (Binnie GD et al. Learning disabilities in epilepsy: neurophysiological aspects. Epilepsia 1990;31(Suppl 4):S2-8; Ped Neur Briefs Feb 1991). Speech delay due to a prelinguistic regression of epileptic origin has been described (Dubois C M et al. Neuropediatrics 2004;35:50-53).

Recovery of cognitive function may occur in children with rolandic-epilepsy, when patients are followed and retested at 5 years after onset, at a time when most have become seizure-free (Lindgren A et al. Epilepsy \& Behavior 2004;5:903-910). 